\title{
Emotional Intelligence, Attachment and Satisfaction with Romantic Relationships among Young Adults: A Brief Report
}

\author{
Leehu Zysberg1*, Gal Kelmer², Limor Mattar² \\ ${ }^{1}$ Gordon College of Education, Haifa, Israel \\ ${ }^{2}$ Tel Hai College, Upper Galilee, Israel \\ Email: *leehuzysberg@yahoo.com, *leehu@gordon.ac.il
}

How to cite this paper: Zysberg, L., Kelmer, G., \& Mattar, L. (2019). Emotional Intelligence, Attachment and Satisfaction with Romantic Relationships among Young Adults: A Brief Report. Psychology, 10, 694-700.

https://doi.org/10.4236/psych.2019.105044

Received: March 19, 2019

Accepted: April 9, 2019

Published: April 12, 2019

Copyright () 2019 by author(s) and Scientific Research Publishing Inc. This work is licensed under the Creative Commons Attribution International License (CC BY 4.0). http://creativecommons.org/licenses/by/4.0/

\begin{abstract}
This study tested the roles emotional intelligence (EI) and attachment styles play in accounting for satisfaction with romantic relationships. It was hypothesized that attachment styles will moderate the association between EI and satisfaction. A sample of 175 young adults living in Israel took measures of EI, attachment styles and satisfaction with romantic relationships. Stepwise regression analysis supported the hypotheses: EI at first did not correlate with satisfaction, but once attachment style was introduced into the model, EI showed a positive association with satisfaction, while both measures of avoidance and anxiety (the two axes of attachment styles) showed a negative association with the same measure. The results are discussed in light of existing evidence and future directions for research and practice are mentioned.
\end{abstract}

\section{Keywords}

Emotional Intelligence, Attachment Style, Romantic Relations, Young Adults

\section{Introduction}

Attaining and maintaining satisfying romantic relationships is considered one of the core characteristics of the well-adjusted adult (Hazan \& Shaver, 1990). Satisfying romantic relationships are considered not just an outcome in its own right but also a factor associated with general well-being, mental health and effective coping with life challenges (Dong, Urista, \& Grundum, 2008; Hazan \& Shaver, 1990; Schimmack et al., 2002). Acknowledging the importance of satisfaction with romantic relations, a large body of research focuses on the antecedents and factors associated with satisfaction with romantic relationships. Researchers ex- 
plored the roles of demographics, personality traits, social pressures and culture in shaping individuals' satisfaction with their romantic relationships (Davis \& Oathout, 1987; Schimmack et al., 2002; Shaver \& Brennen, 1992). Research along these lines revealed that romantic relationships, perhaps even more than other types of long term social interaction, require significant adjustment, adaptation, and coping with various challenges in the emotional and interpersonal domains.

A recent concept of interest in this context is that of Emotional Intelligence (EI). The concept represents two approaches to how people identify, understand and regulate emotions to guide effective behavior patterns in various settings (Salovey \& Mayer, 1990): the first proposes EI is an ability, just like scholastic intelligence, accounting for how effectively individuals process and use emotional information to identify emotions, integrate emotions in reasoning, understand complex emotions and regulate their and other people's behavior accordingly (Mayer \& Salovey, 1993). The second frames EI as an amalgam of personality traits associated with awareness to emotions, intra and inter-personal predispositions, stress management and positive mood maintenance (Petrides \& Furnham, 2001). Although the two approaches vary in how they frame EI, they share core components including: identification and understanding of emotions in self and others, dealing with complex emotional reactions and regulating emotional responses to better adjust to circumstances (Zysberg, 2018). These core characteristics suggest that EI may be positively associated with more effective adjustment and functioning in a broad range of social/interpersonal settings, romantic relationships included. Though this was not often put to empirical test, a preliminary body of evidence supports this direction (Zeidner \& Kaluda, 2008).

However, to test the role EI may play in satisfaction with romantic relationships, one needs to control for the relevant factors often associated with this outcomes. Beyond demographics (such as gender, age, education etc., see for example: Bryan et al., 2001), the literature identifies the concept of attachment style as a psychological factor often associated with how happy people are with their romantic life (Simpson, 1990). Attachments style is a concept describing the underlying assumptions people employ to interpret, understand and manage their relationships and social exchange with others and is often described as ranging from "insecure" to "secure". While insecure individuals tend to be anxious and stressed around managing their associations and interactions with others, secure individuals put trust, and are more open and accepting of the possibility of letting others into their world (Mikulincer, 1995). Naturally, these basic assumptions either encourage and help effective management of interpersonal communication, relation formation and conflict resolution or challenge them, thus having direct impact on any relationship-outcome, let alone satisfaction with romantic associations (Cann et al., 2008).

Some evidence also suggests that EI and attachment styles are correlated: from studies that found direct positive associations between "secure" attachment and most indices of EI (Hamarta, Deniz, \& Saltali, 2009) to evidence showing the in- 
terplay of attachment style alongside EI related abilities in predicting the quality of work relationships (Cherry, Fletcher, \& O'Sullivan, 2013).

Based on the above evidence, we hypothesized that attachment style will moderate the associations between EI and satisfaction with romantic relationships. We tested this model in a sample of young adults in Israel.

\section{Method}

\subsection{Sample}

A convenience sample of 175 young adults who reported being in a romantic relationship for at least 6 months was recruited from communities in Northern Israel. One hundred and seven of them were women and the rest were men. The mean age was 26.24 (s.d. $=3.64$ ); about $50 \%$ had an academic degree, $76 \%$ defined themselves as secular. The mean length of the relationships reported was 36 months (s.d. $=39.34$ ). They all agreed to participate in the study without receiving any tangible benefit or compensation.

\subsection{Measures}

The following measures were used in this study:

Demographic data was collected using a personal questionnaire containing items regarding age, gender, length of the romantic relationship, religiosity and so on.

Satisfaction with the romantic relationship was assessed using the ENRICH (Nurturing Relationship Issues Communication \& Happiness; Olson, Fournier, \& Druckman, 1982). The short Hebrew version of the measure is a 40 item self-report rating scale for various aspects of satisfaction with a romantic relationship which yields a single total score and shows good reliability ranging around 89 (Lavee, 1995).

Emotional Intelligence was assessed using the AVEI (Audio visual test of emotional intelligence; Zysberg, Levy and Zisberg, 2011). This is a computer based test of emotion identification and dealing with emotional complexity (two of the 4 components of ability EI): 27 items present test takers with still pictures of short videos depicting individuals in various social settings, asking the participants to identify the emotions experienced by protagonists in each item. Acceptable reliability and validity were demonstrated in a few studies (e.g.: Raz, Dan, \& Zysberg, 2014).

Attachment style was measured using the ECR (Experience in Close Relationship Scale; Brennan, Clark, \& Shaver, 1998). This 36 item self-report scale assesses two aspects (18 items per aspect) of the axes of anxiety and avoidance allows the classification of respondents to various attachment patterns. We did not use the classification system but rather utilized the two raw scores of the two above described scales. These easily allow the operationalization of a range between insecure (both anxiety and avoidance high) through secure (both at low ranges). A Hebrew version showed high reliability at $.88-.91$ and adequate con- 
struct validity (Mikulincer \& Florian, 2001).

\subsection{Procedure}

Participants were approached on social media including Facebook, and online groups. They were asked to participate in a study of romantic relationships. Links to online questionnaires were supplied and the system was designed so that no identifying email address or IP address was retained, so once submitted, the information could not be traced to a specific person. Completing the questionnaires online took about 15 minutes.

The resulting database was analyzed using SPSS v.24 (IBM, 2016).

\section{Results}

\section{Descriptive Statistics}

Before testing the study hypothesis we calculated descriptive statistics for the main variables in our study. These are summarized in Table 1 herein.

No ceiling of floor effects was evident in our data, and reliabilities ranged acceptable to high. We therefore proceeded to test our hypothesis using a step-wise regression analysis: In the first step we entered EI as the only predictor and used the total satisfaction score as criterion. We then added the attachment scores and the interaction terms between EI and each of the attachment measures to the predictors. The results are summarized in Table 2.

To preclude the possibility of collinearity we tested the associations between the predictors and found no association between EI and the attachment scores.

The results show that in the first step EI did not associate with the satisfaction

Table 1. Descriptive statistics $(n=175)$.

\begin{tabular}{cccccc}
\hline Variable name & Mean & SD & Min & Max & Cronbach's Alpha \\
\hline EI & 16.87 & 4.17 & 0 & 24 & .71 \\
Attachment - Avoidance & 3.26 & .84 & 1.56 & 5.33 & .87 \\
Attachment - Anxiety & 3.15 & 1.06 & 1.06 & 6.11 & .91 \\
Satisfaction & 156.93 & 20.71 & 70 & 192 & .87
\end{tabular}

Table 2. A summary of a regression analysis using EI and attachment style score to account for the variance in satisfaction with romantic relations $(n=175)$.

\begin{tabular}{cccccccccc}
\hline & \multicolumn{3}{c}{ Step1 } & \multicolumn{3}{c}{ Step2 } & \multicolumn{3}{c}{ Step 3 } \\
\hline & B & SE & Beta & B & SE & Beta & B & SE & Beta \\
\hline EI & .13 & .08 & .13 & .19 & .08 & $.18^{\star *}$ & .19 & .08 & $.17^{\star}$ \\
Avoidance & & & & -.29 & .09 & $-.28^{* *}$ & -.28 & .07 & $-.28^{\star *}$ \\
Anxiety & & & & -.24 & .07 & $-.24^{* *}$ & -.24 & .07 & $-.24^{* *}$ \\
EI*Avoidance & & & & & & & -.04 & .08 & -.03 \\
EI*Anxiety & & & & & & & .03 & .09 & .03 \\
\hline
\end{tabular}

${ }^{\star} p<.05,{ }^{* *} p<.01$. 
grade, however once entering the attachment style grades both them and the EI grade associated with satisfaction. Both measures of attachment showed negative associations with satisfaction and EI showed a positive association with the same outcome measure. Interaction terms added to the analysis did not show any significant association with satisfaction.

\section{Discussion}

Preliminary evidence suggests that IE may play a significant role in accounting for satisfaction with romantic relationships (e.g.: Zeidner \& Kaluda, 2008). We added to the discussion of this relatively novel issue by re-examining the potential association between a test format measure of EI and satisfaction with romantic relationships while hypothesizing that attachment style, often found to underlie the nature of human relations in a myriad of contexts, may moderate this association.

The results, based on a sample of 175 young adults from Israel, who reported being in a committed romantic relationship for at least 6 months at the time of the study, supported the moderation model: no direct association between EI and satisfaction was found, however, once both measures of attachment style were inserted into the analyses, both them and the measure of EI associated with satisfaction (the attachment measures representing avoidance and anxiety showed a negative association and EI a positive association with satisfaction).

These results echoed the existing literature on attachment style and satisfaction with romantic relationships, or relationships in general (see for example: Feeney \& Noller, 1990; Leiter, Day, \& Price, 2015). A slightly more novel and surprising finding is the moderated association between EI and satisfaction with romantic relationships: studies lead us to expect that EI will also show direct association with satisfaction with relationships since the concept is often associated with social skills, regulation and adjustment in interpersonal contexts (Zeidner, Matthews, \& Shemesh, 2016). Our findings suggest that the association between EI and satisfaction with romantic relationships emerges only after attachment styles are brought into consideration. This pattern may shed light on how EI associates with satisfaction of romantic (and perhaps other) relationships: EI may serve as a coping resource, an ability to use emotional information to shape social behavior more effectively, thus raising satisfaction of relations. However, attachment styles may either mask this ability or highlight it. While secure style may enhance the effective use of emotions in relationships, insecure style may mask this ability.

When considering the results reported herein, one should bear in mind the limitations of the study: this was a correlational study, thus, preventing us from drawing any conclusion regarding causation or chronology of the effects. In other words, we at this point cannot say what comes first: attachment style or EI? We sampled young Israelis: culture may play a role in their perception of romantic relations, especially since we did not focus primarily on married 
couples. The use of a combination of self-report and test format measures is beneficiary to the validity of the results, however, using behavioral-observational measures may be of added value in future studies.

Even after considering the limitations, this study presents a first of its kind model and evidence to the role of attachment style and EI in satisfaction with romantic relationships in young adults. The results may also shed new light on how EI works to account for satisfaction with romantic (and maybe other) relationships. Future studies may wish to examine the model in various samples from diverse populations, examine the longitudinal effects of personal factors such as EI, attachment style and more on relationship quality indices in the long run. Such insights may both enrich our theoretical understanding of this central issue in most peoples' lives and may point the way to future interventions and program aimed at increasing individuals' and couple's enjoyment of the most elusive thing of all true love.

\section{Conflicts of Interest}

The authors declare no conflicts of interest regarding the publication of this paper.

\section{References}

Brennan, K. A., Clark, C. L., \& Shaver, P. R. (1998). Self-Report Measurement of Adult Attachment: An Integrative Overview.

Bryan, L., Fitzpatrick, J., Crawford, D., \& Fischer, J. (2001). The Role of Network Support and Interference in Women's Perception of Romantic, Friend, and Parental Relationships. Sex Roles, 45, 481-499. https://doi.org/10.1023/A:1014858613924

Cann, A., Norman, M. A., Welbourne, J. L., \& Calhoun, L. G. (2008). Attachment Styles, Conflict Styles and Humour Styles: Interrelationships and Associations with Relationship Satisfaction. European Journal of Personality: Published for the European Association of Personality Psychology, 22, 131-146. https://doi.org/10.1002/per.666

Cherry, M. G., Fletcher, I., \& O'Sullivan, H. (2013). The Influence of Medical Students' and Doctors' Attachment Style and Emotional Intelligence on Their Patient-Provider Communication. Patient Education and Counseling, 93, 177-187. https://doi.org/10.1016/j.pec.2013.05.010

Davis, M. H., \& Oathout, H. A. (1987). Maintenance of Satisfaction in Romantic Relationships: Empathy and Relational Competence. Journal of Personality and Social Psychology, 53, 397. https://doi.org/10.1037/0022-3514.53.2.397

Dong, Q., Urista, M. A., \& Gundrum, D. (2008). The Impact of Emotional Intelligence, Self-Esteem, and Self-Image on Romantic Communication over MySpace. CyberPsychology \& Behavior, 11, 577-578. https://doi.org/10.1089/cpb.2007.0154

Feeney, J. A., \& Noller, P. (1990). Attachment Style as a Predictor of Adult Romantic Relationships. Journal of Personality and Social Psychology, 58, 281. https://doi.org/10.1037/0022-3514.58.2.281

Hamarta, E., Deniz, M., \& Saltali, N. (2009). Attachment Styles as a Predictor of Emotional Intelligence. Educational Sciences: Theory and Practice, 9, 213-229.

Hazan, C., \& Shaver, P. R. (1990). Love and Work: An Attachment-Theoretical Perspective. Journal of Personality and Social Psychology, 59, 270. 
https://doi.org/10.1037/0022-3514.59.2.270

IBM (2016). IBM SPSS Statistics for Windows, Version 24.0. Armonk, NY: IBM Corp.

Lavee, Y. (1995). The Israeli Marital Quality Scale (I-MQS): Clinical and Research Application. The 25th Annual Conference of the Israeli Psycological Association, Ben Gurion University, Beer-Sheva, Israel.

Leiter, M. P., Day, A., \& Price, L. (2015). Attachment Styles at Work: Measurement, Collegial Relationships, and Burnout. Burnout Research, 2, 25-35. https://doi.org/10.1016/j.burn.2015.02.003

Mayer, J. D., \& Salovey, P. (1993). The Intelligence of Emotional. Intelligence, 17, 433-442. https://doi.org/10.1016/0160-2896(93)90010-3

Mikulincer, M. (1995). Attachment Style and the Mental Representation of the Self. Journal of Personality and Social Psychology, 69, 1203. https://doi.org/10.1037/0022-3514.69.6.1203

Mikulincer, M., \& Florian, V. (2001). Attachment Style and Affect Regulation: Implications for Coping with Stress and Mental Health. In Handbook of Social Psychology: Interpersonal Processes (pp. 535-557). Cambridge: Blackwell.

Olson, D. H., Druckman, J. M., \& Fournier, D. (1982). Enrich. University of Minnesota, Family Social Science.

Petrides, K. V., \& Furnham, A. (2001). Trait Emotional Intelligence: Psychometric Investigation with Reference to Established Trait Taxonomies. European Journal of Personality, 15, 425-448. https://doi.org/10.1002/per.416

Raz, S., Dan, O., \& Zysberg, L. (2014). Neural Correlates of Emotional Intelligence in a Visual Emotional Oddball Task: An ERP Study. Brain and Cognition, 91, 79-86. https://doi.org/10.1016/j.bandc.2014.09.003

Salovey, P., \& Mayer, J. D. (1990). Emotional Intelligence. Imagination, Cognition and Personality, 9, 185-211. https://doi.org/10.2190/DUGG-P24E-52WK-6CDG

Schimmack, U., Radhakrishnan, P., Oishi, S., Dzokoto, V., \& Ahadi, S. (2002). Culture, Personality, and Subjective Well-Being: Integrating Process Models of Life Satisfaction. Journal of Personality and Social Psychology, 82, 582. https://doi.org/10.1037/0022-3514.82.4.582

Shaver, P. R., \& Brennan, K. A. (1992). Attachment Styles and the "Big Five" Personality Traits: Their Connections with Each Other and with Romantic Relationship Outcomes. Personality and Social Psychology Bulletin, 18, 536-545. https://doi.org/10.1177/0146167292185003

Simpson, J. A. (1990). Influence of Attachment Styles on Romantic Relationships. Journal of Personality and Social Psychology, 59, 971. https://doi.org/10.1037/0022-3514.59.5.971

Zeidner, M., \& Kaluda, I. (2008). Romantic Love: What's Emotional Intelligence (EI) Got to Do with It? Personality and Individual Differences, 44, 1684-1695. https://doi.org/10.1016/j.paid.2008.01.018

Zeidner, M., Matthews, G., \& Shemesh, D. O. (2016). Cognitive-Social Sources of Wellbeing: Differentiating the Roles of Coping Style, Social Support and Emotional Intelligence. Journal of Happiness Studies, 17, 2481-2501. https://doi.org/10.1007/s10902-015-9703-z

Zysberg, L. (2018). Emotional Intelligence and Health Outcomes. Psychology, 9, 2471. https://doi.org/10.4236/psych.2018.911142

Zysberg, L., Levy, A., \& Zisberg, A. (2011). Emotional Intelligence in Applicant Selection for Care-Related Academic Programs. Journal of Psychoeducational Assessment, 29, 27-38. 